\title{
Preface
}

\section{Innovations in Thoracic Surgery}

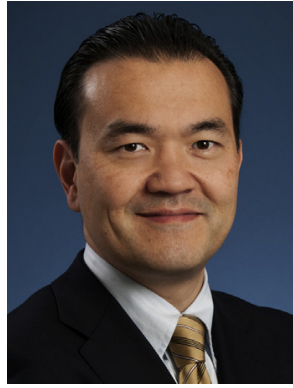

Kazuhiro Yasufuku, MD, PhD Editor
Since the first successful one-stage pneumonectomy for lung cancer performed by Dr Evarts Graham in 1933, modern thoracic surgery has evolved significantly. Various technology introduced over time has advanced thoracic surgery from open procedures to minimally invasive surgeries. Perhaps one of the most significant developments in thoracic surgery has been the advent of video-assisted thoracoscopic surgery (VATS). The invention of the Charged Coupled Device Image Sensor has allowed miniaturization of surgical cameras, thus allowing the use of the high-definition thoracoscope during minimally invasive surgeries. New developments in surgical instruments, stapling devices, and energy devices have allowed surgeons to perform more advanced and complex VATS procedures. Surgical robotic technology has also emerged as an approach to a different dimension of minimally invasive surgery.

Development of technology in endoscopy has set a new standard for ultraminimally invasive thoracic procedures. Now, incisionless endoscopic surgeries can be performed for benign esophageal disease and to manage patients with early-stage esophageal cancer. Novel bronchoscopic technologies, including navigational bronchoscopy and endobronchial ultrasound, have opened up alternatives to invasive diagnostic/ therapeutic procedures for lung cancer. There are various types of technology that have been developed for bronchoscopic lung volume reduction for the management of patients with chronic obstructive pulmonary disease. Endoscopic/bronchoscopic procedures are now being utilized in combination with advanced imaging during VATS for management of thoracic malignancies.

In this issue of Thoracic Surgery Clinics, innovations in thoracic procedures are reviewed with contributions from the experts in the field. The different technical aspects of new technologies currently available for thoracic surgeons are discussed. In addition, new technologies on the horizon, including nanotechnology, isolated lung perfusion technology, and bioengineered organs, are introduced. I would like to thank all of the contributing authors for their expertise and contributions to this issue.

Kazuhiro Yasufuku, MD, PhD University Health Network Interventional Thoracic Surgery Program

Division of Thoracic Surgery Toronto General Hospital

University of Toronto 200 Elizabeth Street, 9N-957 Toronto, ON M5K 2C4, Canada

E-mail address: kazuhiro.yasufuku@uhn.ca 